\title{
Inequalities in Specialist Hand Surgeon Distribution Across the United States
}

Background: Unequal access to hospital specialists for emergency care is an issue in the United States (US). The objective of this study was to describe the geographical distribution of surgeons with a Subspecialty Certificate in Surgery of the Hand (SCSH) and associated factors in the US.

Methods: Geographic distribution of surgeons holding a SCSH and hand surgery fellowship positions were identified from the American Board of Medical Specialties Database and the literature (2013), respectively. State-level population and per capita income were ascertained using US census data. Variations in hand trauma admissions were determined using Healthcare Cost Utilization Project national/state inpatient databases. Risk-adjusted generalized linear models were used to assess independent association between hand surgeon density and hand trauma admissions density, fellowship positions density, and per capita income.

Results: Among 2,019 specialist hand surgeons identified; 72.1\% were orthopedic (OS), 18.3\% plastic (PS), and 9.6\% general (GS) surgeons. There were 157 hand surgery fellowship positions nationwide. There were 149,295 annual hand trauma admissions. The national density of specialist hand surgeons and trauma admission were 0.6 and 47.6 , respectively. The density of specialist hand surgeons varied significantly between states. State-level variations in density of surgeons were independent and significantly associated with median per capita income $(p<0.001)$ and with density of fellowships $(\mathrm{p}=0.014)$.

Conclusion: Specialist hand surgeons are distributed unevenly across the US. State-level analyses suggest that states with lower per-capita incomes may be particularly underserved, which may contribute to regional disparities in access to emergency hand trauma care. 


\section{INTRODUCTION}

The lack of specialty emergency care across hospitals in the United States is increasingly recognized as a major healthcare problem. ${ }^{1,2}$ The Institute of Medicine referred to this problem as one of the most troubling aspects of emergency care system ${ }^{3}$ and one of the greatest challenges for emergency departments (EDs) has been the provision of on-call specialist coverage. ${ }^{2}$ The consequences of inadequate specialist availability include delayed treatment, emergency department overcrowding, long patient transfer times, and unsustainable pressure on specialist referral centers. ${ }^{4}$ Patients may further receive sub-optimal treatment in the absence of specialist advice.

This crisis is particularly severe in orthopedic and plastic surgery ${ }^{1,2,5}$; and hand surgery is among the specialties struggling to provide adequate on-call coverage ${ }^{1,2}$, despite monetary incentives. ${ }^{2}$ One cross-sectional survey found that $80 \%$ of ED directors reported inadequate hand surgery coverage at their institutions. ${ }^{5}$ In Florida, only $38.5 \%$ hospitals treating injured patients have a specialist hand surgeon available at all times ${ }^{6}$ and, although level I trauma centers in Tennessee are mandated to have a hand specialist available out of hours, only $25 \%$ fulfilled this requirement in $2013 .^{7}$

A previous effort made by the American Society for Surgery of the Hand Task Force described the national number of hand surgery fellowships, applicants, graduates, and board certification status (Subspecialty Certificate in Surgery of the Hand [SCSH]). However, this study focused on the typical career pathways of hand surgeons rather than the actual distribution of hand surgeons across the United States. ${ }^{8}$ The availability of specialist hand surgeons managing acute hand injuries is directly related to the proximity of these surgeons to injured patients and therefore a comprehensive assessment of the distribution of specialist hand surgeons is necessary. In order to understand the relationship between training, surgeon availability, and population need, this study 
sought to describe the geographic distribution of surgeons with a SCSH and associated factors in the US.

\section{METHODS}

The analysis was conducted as a state-level ecological study based on data collected from a range of administrative sources. The study was approved by the Partners HealthCare Institutional Review Board (2015P000745/BWH). Factors included geographic distributions of: the number of hand surgeons per 100,000 population (hand surgeon density), the number of fellowship positions per 100,000 population (fellowship position density), median per capita income, and the number of hand trauma admissions per 100,000 population (hand trauma admissions density) by state and on a national scale.

\section{Subspecialist Hand Surgeons}

Data published by the American Board of Medical Specialties (ABMS) were used to identify hand surgeons holding a SCSH and their primary specialty in all states (2013). ${ }^{9}$ The ABMS previously compiled this resource from state-level data on more than 800,000 specialty physicians across the US. Subspecialist hand surgeons serving in the military (2) or practicing outside the US (5) were excluded due to the inability to locate them within the US territories. Beyond SCSH surgeons, the work presented in this paper further quantified the number of physicians practicing as hand surgeons but without a current SCSH. This was achieved through a search of board of medicine websites for 18 states, which have public data on physicians' selfreported specialties and location of practice. To be included, surgeons should have been currently practicing, board certified within their state, and have declared a subspecialty interest in hand surgery. This search was conducted on $27^{\text {th }}$ April 2015. 
For the purposes of this paper, "subspecialist hand surgeons" describes board-certified surgeons holding a valid SCSH.

\section{Hand Surgery Fellowships}

Data on fellowship positions has been previously described by Goldfarb et al. ${ }^{8}$ Results from this assessment of 78 hand surgery fellowship programs across the US (2013) were used to plot training availability by state.

\section{Population and Income Estimates}

The US Census projections were used to obtain 2012 population estimates. ${ }^{10}$ Per capita income estimates at the state level were obtained from the 2009-2013 American Community Survey 5Year Estimates. ${ }^{11}$

\section{Incidence of Hand Trauma}

The Healthcare Cost and Utilization Project Nationwide Inpatient Sample (HCUP-NIS) was queried to determine the number of hand trauma admissions nationally. In the same fashion, all the Healthcare Cost and Utilization Project State Inpatient Databases (HCUP-SIDs) available $(37 / 50,74 \%)$ were queried to determine the number of hand trauma admissions by state. These queries were performed through the Healthcare Cost Utilization Project website (HCUPnet). ${ }^{12}$ Patients were identified based on all-listed International Classification of Diseases, $9^{\text {th }}$ revision, Clinical Modification (ICD-9-CM) diagnosis codes corresponding to hand trauma (Appendix 1). Data were extracted for the most recent year available (2012). The most recent data reported for New Hampshire was from 2009. HCUPnet also allowed to obtain the percentage of patients with primary hand injury diagnoses codes that were transferred to other hospitals, which was performed to discern both referral patterns and care pathways.

The NIS is the largest publicly available all-payer inpatient health care database in the United States and it provides national weighted estimates of hospital inpatient stays based on 
discharges. ${ }^{13}$ The SIDs include inpatient all-payer discharge records from community hospitals by State. ${ }^{14}$

\section{Statistical Analysis}

Geographical distributions of the number of subspecialist hand surgeons and fellowship positions were explored using descriptive statistics. To correct for the size of population per state, density (per 100,000 population) of subspecialist hand surgeons, fellowship positions, and hand trauma admissions were calculated. Intensity maps were created to illustrate these values. In order to assess independent association between hand surgeon density (main outcome) and hand trauma admissions density, fellowship positions density, and per capita income (covariates), risk-adjusted generalized linear models followed by post-estimation calculation of average marginal effects were used to yield predicted mean differences and $95 \%$ confidence intervals $(95 \% \mathrm{CI})$. Since hand surgeon density was highly skewed, and in order to account for the non-normal nature of this distribution, modified Park tests were used to determine the appropriate modeling strategy, which corresponded to a gamma distribution. Subanalysis testing the association between density of hand trauma admission and per capita income were performed. Intensity maps were created using SPSS 22 (IBM, New York, NY) and statistical analyses performed in Stata 12.0 (College Station, TX). Two-sided p-values $<0.05$ were considered statistically significant.

\section{RESULTS}

There were 2,019 subspecialist hand surgeons; $72.2 \%$ were primarily OS, $18.3 \%$ PS, and 9.6\% GS. Subspecialist hand surgeons represented $5.9 \%$ of all board-certified OS, $6.0 \%$ PS, and $0.6 \%$ GS. The primary specialty of subspecialist hand surgeons varied widely by state: only OS were represented in the District of Columbia, Hawaii, Maine, Montana, and South Dakota (Figure 1a). There was no PS representation in Alaska, Delaware, New Mexico, or North Dakota; and no GS 
in Kansas, Vermont, or Wyoming. In terms of self-declared subspecialist hand surgeons, there were 1,321 with active licenses in 18 states (the only states that report this data). In these same states, there were 967 (73.2\%) surgeons holding a SCSH.

The density of subspecialist hand surgeons varied drastically between states (Figure 2a). Overall, there were 0.64 hand surgeons per 100,000 population, although the density was over three times higher in the top five states when compared with the bottom five. Visual inspection of the data revealed five distinct zones where densities were comparable. The first zone (well above the mean) was found above 0.95 ; the second zone (above the mean) found in the $0.85-0.80$ range; the third zone (mean) in the $0.70-0.55$ range; the fourth zone (below the mean) in the $0.50-0.40$ range; and the fifth zone (well below mean) below 0.40 (Figure $2 \mathrm{~b}$ ).

There were 157 hand surgery fellowship positions offered in 30 states, $82.8 \%$ for OS, $12.1 \%$ for PS, and 5.1\% for GS. In half of the states (15/30) there were available only OS-run fellowships, and Kentucky was the only state with a GS-run fellowship (Figure 1b). The fellowship density also varied remarkably between states (Figure 2c). Overall, there were 0.05 fellowship positions per 100,000 but the density in the top five states was almost ten times higher than those in the bottom five. Visual inspection of the data revealed five distinct distribution zones where densities of fellowship positions per 100,000 population per state were grouped. The first zone (well above the mean) above 0.12 ; the second zone (above the mean) in the $0.10-0.80$ range; the third zone (mean) in the $0.70-0.50$ range; the fourth zone (below mean) in the $0.40-0.30$; and the fifth zone (well below mean) below 0.40 .

There were 149,295 hand trauma admissions across the US in 2012, or 46.8 admissions per 100,000 population (Figure 2e). At the state level, hand trauma admissions (per 100,000 population) in the top five states were twice that of the bottom five. Visual inspection of the data also revealed a more uniform pattern with the exception of high-density outliers states with above 55 admissions per 100,000 population (Figure $2 \mathrm{f}$ ). There are 14 states that are not represented, as 
they do not report these data through HCUPnet. Since hand trauma admissions were used as a marker of the incidence of severe trauma per state, patients' demographic and clinical characteristics between states were not analyzed but they are included in a supplemental file (Supplemental file).

In risk-adjusted models, there was an independent positive association of subspecialist hand surgeon density and per capita income at the state level. For every $\$ 10,000$ increase in per capita income, the risk-adjusted predicted mean number of subspecialist hand surgeons per 100,000 population increased by $3.36(95 \% \mathrm{CI} 1.78-4.50 ; \mathrm{p}<0.001)$. There was also an independent positive association of subspecialist hand surgeon density and fellowship density. For every unit increase in fellowship positions per 100,000 population, the risk-adjusted predicted mean number of subspecialist hand surgeons per 100,000 population increased by 1.07 (95\% CI $0.21-1.92$; $\mathrm{p}=0.014)$. However, there was no significant association of subspecialist hand surgeon density and density of trauma-related admissions in risk-adjusted models $(\mathrm{p}=0.142)$, and there was no significant association between per capita income and the density of trauma-related admissions in non-risk-adjusted models $(\mathrm{p}=0.752)$.

\section{DISCUSSION}

Subspecialist hand surgeons are distributed unequally across the United States. Although the reasons for unequal distribution of subspecialist hand surgeons are unknown, we conducted a risk-adjusted analysis to explore associations with potentially relevant factors. Specifically, we looked for independent associations between hand surgeon density and per capita income, hand trauma clinical need and availability of specialist hand fellowships; and found that per-capita income was the only factor independently associated with higher density of hand surgeons.

There was a significant positive association between subspecialist hand surgeon density and per capita income at the state level. This finding is consistent with previous work that found poor counties in Tennessee are underserved by hand surgeons. ${ }^{7}$ It has also has also been demonstrated 
in other healthcare systems: in Germany, every percent change in the prevalence of people with comprehensive health insurance is associated with a $2.1 \%$ increase in hospital specialist density. ${ }^{15}$ Furthermore, it is likely that this pattern contributes to the difficulties some hospitals face in accessing specialist hand services. ${ }^{1,5,6}$ Previous research conducted among 56 Oregon hospitals showed that chief executive officers faced difficulties in on-call coverage despite an $84 \%$ increase in the annual stipend for promoting specialist on-call coverage, including hand surgeons. ${ }^{2}$ Rudkin et al. ${ }^{1}$ in a statewide analysis of EDs in California identified hand surgery as one of the specialties, among surgical and non-surgical, that had trouble with on-call coverage greater than $50 \%$ of the time. Other contributory factors may include physician preference for elective ambulatory care without on-call responsibility ${ }^{6}$ and socioeconomic incentives. ${ }^{7}$ Financial incentives might play a role in surgeon density, and government payers are likely a significant contributor in low socioeconomic status states. Further studies should consider subspecialist hand surgeon income in well-served and under-served states to determine whether this is a factor driving distribution. It has been previously shown that physicians aggregate in specific regions, with one study reporting that specialists are less evenly distributed around the United States than generalists. ${ }^{16}$ The Council on Graduate Medical Education has described this issue as "the central paradox of the American health care system: shortages amid surplus". ${ }^{17}$ This relationship raises the issue of geographical access to care and may contribute to healthcare disparities. ${ }^{18}$

The number of hand-trauma-related hospital admissions was investigated to determine whether the distribution of subspecialist hand surgeons was obviously explained by clinical need. However, in risk-adjusted models there was no independent association between hand surgeons and hand trauma admissions, which suggests that the trauma burden cannot explain this phenomenon. This lack of association is also important because it is further evidence that injured patients in low-income states are underserved by subspecialist hand surgeons. The ideal finding would have been a significant positive association between hand injury admissions and 
availability of subspecialists. Additionally, in subanalysis testing the association of trauma admissions with per capita income, there was no significant association, suggesting that handrelated admissions in high-income states is not different from those with lower income and therefore not explaining the distribution of subspecialist hand surgeons.

The results presented do not show that subspecialist hand surgeons provide superior care, even for patients with complex hand injuries. Nonetheless, the distribution zones of hand surgeons and fellowship densities provided in the present study could be used as thresholds in future research. Further research should use these thresholds when examining associations between well-served and/or underserved regions with surgical outcomes as previously done for general surgery, where it was shown that the supply of surgeons does affect access to surgical services, which in turn has an impact on surgical complications. ${ }^{19}$

As hand surgery trainees are part of the specialty's workforce, the present study also analyzed the independent association of the density of hand surgeons and fellowship positions. There was a significant positive association between the density of subspecialist hand surgeons and subspecialty fellowship position density. It is likely that these factors are causally related in a circular fashion. Stronger hand fellowships tend to occur where more surgeons congregate; and large practices are likely to benefit from the support of a fellowship. Hand surgeons and their impetus are needed in various underserved areas to stablish new fellowships in these regions.

A number of limitations should be recognized when interpreting these findings. First, the analyses presented are based on hand surgeons holding a SCSH. Detailed requirements for obtaining the SCSH are described elsewhere ${ }^{8}$ but include a minimum number of logged operative cases, a clinical practice predominantly in hand surgery, and the satisfactory completion of a multiple choice examination $^{20}$. Maintenance of certification involves repeat examination on a ten-yearly cycle. It is conceivable that a proportion of practicing hand surgeons have also previously held a SCSH which has since expired and not been renewed. Nevertheless, surgeons holding a SCSH 
were used in the analysis because it provides a useful threshold for identifying those that have received additional subspecialty training, reached a minimum threshold, and maintained a continued commitment to hand surgery; and because it permitted the reliable identification of hand surgeons per state. ${ }^{8}$ In addition, our investigation of self-reported hand surgeons suggests that those holding a SCSH represent most (73.2\%) of the hand surgery workforce, which is comparable to the two-thirds (63\%) found by the American Society for Surgery of the Hand Task Force from 2000-2006. ${ }^{8}$ Second, we used inpatient hand trauma admissions as a general marker of state hand trauma burden. This inevitably excludes patients treated in ambulatory settings, e.g. by a primary care practitioner or emergency room physician. However, these data did capture hand injuries that were severe and/or complex enough to require a hospital admission and permitted equivalent comparisons between states. As this methodology was applied consistently across all states in our study, it should serve as a marker for the overall burden of severe hand injuries. We considered narrowing the scope of analysis to a small number of the most complex hand-related trauma diagnosis codes to improve focus of the study, but found that there were too little data unless grouped codes were used. Finally, we were not able to exclude transferred patients as HCUPnet does not allow to discern these patients when querying for all-listed diagnoses. However, analysis of patients with a primary hand injury diagnosis in the NIS found that the national inter-hospital transfer rate for these patients is low $(0.78 \%)$.

This study has shown that subspecialist hand surgeons are unequally distributed across the US and that this distribution is correlated with median per capita income. States with lower per-capita incomes may be particularly underserved, which may contribute to regional disparities in access to emergency hand trauma care. Future studies are necessary to determine the reasons for this distribution of subspecialist hand surgeons across the US States and whether this leads to worse care and/or impaired access to specialist hand services for patients in low-income states. 


\section{REFERENCES}

1. Rudkin SE, Oman J, Langdorf MI, et al. The state of ED on-call coverage in California. The American journal of emergency medicine. Nov 2004;22(7):575581.

2. McConnell KJ, Newgard CD, Lee R. Changes in the cost and management of emergency department on-call coverage: evidence from a longitudinal statewide survey. Annals of emergency medicine. Dec 2008;52(6):635-642.

3. Institute of Medicine. Hospital-Based Emergency Care: At the Breaking Point. Washington, DC2006.

4. Southard P. 2003 "clarification" of controversial EMTALA requirement for $24 / 7$ coverage of emergency departments by on-call specialists, significant impact on trauma centers. Journal of emergency nursing: JEN : official publication of the Emergency Department Nurses Association. Dec 2004;30(6):582-583.

5. Rao MB, Lerro C, Gross CP. The shortage of on-call surgical specialist coverage: a national survey of emergency department directors. Academic emergency medicine : official journal of the Society for Academic Emergency Medicine. Dec 2010;17(12):1374-1382.

6. Caffee H, Rudnick C. Access to hand surgery emergency care. Annals of plastic surgery. Feb 2007;58(2):207-208.

7. Anthony JR, Poole VN, Sexton KW, et al. Tennessee emergency hand care distributions and disparities: Emergent hand care disparities. Hand (New York, N.Y.). Jun 2013;8(2):172-178.

8. Goldfarb CA, Lee WP, Briskey D, Higgins JP. An American Society for Surgery of the Hand (ASSH) task force report on hand surgery subspecialty certification and ASSH membership. The Journal of hand surgery. Feb 2014;39(2):330-334.

9. American Board of Medical Specialties. 2013-2014 ABMS Board Certification Report. Chicago, IL2014.

10. United States Census Bureau. 2012 Population Estimates. 2014; http://www.census.gov/popest/data/historical/2010s/vintage 2012/state. $\underline{\mathrm{html}}$. Accessed April 30, 2015.

11. United States Census Bureau. 2009-2013 American Community Survey 5Year

Estimates. 2014; http://factfinder.census.gov/faces/tableservices/isf/pages/productview.xht ml?pid=ACS 13 5YR DP03\&prodType=table. . Accessed April 30, 2015.

12. Agency for Healthcare Research and Quality. HCUPnet. 2015; http://hcupnet.ahrq.gov. Accessed May 21, 2014.

13. Agency for Healthcare Research and Quality. Overview of the National (Nationwide) Inpatient Sample (NIS). 2015; http://www.hcupus.ahrq.gov/nisoverview.jsp. Accessed Apr 1, 2015.

14. Agency for Healthcare Research and Quality. Overview of the State Inpatient Databases (SID). 2015; http://www.hcup-us.ahrq.gov/nisoverview.jsp. Accessed Apr 1, 2015. 
15. Sundmacher L, Ozegowski S. Regional distribution of physicians: the role of comprehensive private health insurance in Germany. The European journal of health economics : HEPAC : health economics in prevention and care. Apr 30 2015.

16. Neuwahl S, Ricketts TC, Thompson K. Geographic distribution of general surgeons: comparisons across time and specialties. Bulletin of the American College of Surgeons. Sep 2011;96(9):38-41.

17. Council on Graduate Medical Education. Physician distribution and health care challenges in rural and inner-city areas. Washington, DC1998.

18. Hsia R, Shen YC. Possible geographical barriers to trauma center access for vulnerable patients in the United States: an analysis of urban and rural communities. Archives of surgery. Jan 2011;146(1):46-52.

19. Neuwahl S, Ricketts T, Holmes M. The impact of general surgeon supply on the risk of appendiceal rupture in North Carolina. Annals of surgery. May 2014;259(5):910-915.

20. Stern PJ. Subspecialty certification in hand surgery. Clinical orthopaedics and related research. Aug 2006;449:165-168. 


\section{FIGURE LEGENDS}

Figure 1. Stacked percent contribution to overall number of subspecialist hand surgeons in each state according to primary surgical specialty (a). Stacked percent contribution to overall number of fellowships available in each state according to primary surgical specialty of the program (b).

Labels:

a) upper image

b) Lower image

Footnote: $\left(^{*}\right)$ represents United States statistics.

Figure 2. Distribution by state of subspecialist hand surgeons per 100,000 population (a and b); hand surgery fellowship positions per 100,000 population (c and d); and hand trauma per 100,000 population in the United States (e and f).

Labels:

a) Upper left image

b) Upper right image

c) Middle left image

d) Middle right image

e) Lower left image

f) Lower right image

Footnote: $\left(^{*}\right)$ represents United States statistics. 